\title{
Impacto da cárie dentária na qualidade de vida de adultos residentes no entorno de Belo Horizonte, MG, Brasil
}

\author{
Impact of dental caries on quality of life among adults resident \\ in greater Belo H orizonte, State of M inas Gerais, Brazil
}

Simone de M elo Costa ${ }^{1}$

Mara Vasconcelos ${ }^{2}$

M auro Henrique N ogueira Guimarães Abreu ${ }^{2}$
${ }^{1}$ Universidade Estadual de M ontes Claros, Unimontes. Campus Universitário Professor Darcy Ribeiro, Vila M auricéia. 39401-089 Montes Claros M G. smelocosta@gmail.com ${ }^{2}$ Departamento de Odontologia, Universidade Federal de M inas Gerais.
Abstract This paper discusses the impact of dental carries on quality of life. The aim was to assess the quality of life of adults in greater Belo Horizonte (state of M inas Gerais, Brazil) in accordance with the prevalence and severity of dental caries. A domicile-based cross-sectional study was carried out with cluster sampling. Data collection involved the DM FT index and WHOQOL-BREF questionnaire. Theprevalence of dental caries was defined based on the presence of at least one carious tooth $(D \geq 1)$ and greater severity was defined as DMFT $\geq 25$. Among the 1138 adults studied, 461 had at least one carious tooth. Higher quality of life values were found among individuals without dental caries and those with lesser severity of caries. A greater number of carious teeth and higher DM FT index denoted a reduction in quality of life $(p<0.001)$. N egative correlations between dental caries and quality of life were determined in the psychological, social and environmental domains. In conclusion, dental caries exerts a negative impact on the quality of life of adults. Subjective indicators can contribute in qualifying clinical information on oral health.

Key words Dental caries, Quality of life, Epidemiology
Resumo 0 artigo discuteo impacto da cárie dentária na qualidade devida das pessoas. 0 objetivo foi avaliar a qualidade de vida de adultos moradores no entorno de Belo H orizonte, M inas Ge rais, Brasil, conforme a prevalência e a severidade de cárie dentária. Trata-se de estudo transversal, de basedomiciliar realizado a partir de amos tragem por conglomerados. Os indicadores utilizados na coleta de dados foram o índice de cárie CPOD e a Escala WHOQOL-bref. A prevalência de cárie foi definida a partir da presença de pelo menos um dentecariado $(C \geq 1)$ ea maior gravidade pelo CPOD $\geq 25$. A população examinada fo de 1138 adultos, sendo que 461 tinham pelo menos um dente cariado. H ouve melhores índices de qualidade de vida para os grupos de pessoas sem cárie e com menor gravidade de cárie. À medida que o número de dentes cariados e 0 CPOD aumentam a qualidadedevida diminuí ( $p<0,001$ ) A correlação negativa entre cárie e qualidade de vida foi verificada nos domínios psicológico, relações sociais e meio ambiente. Concluiu-se que a cárie dentária resulta em impacto negativo na qualidade de vida de adultos. Os indicadores subjetivos contribuíram para qualificar as informações clínicas de saúde bucal.

Palavras-chave Cárie dentária, Qualidade de vida, Epidemiologia 
Introdução

A cárie dentária tem sido considerada, em nível mundial, como uma das enfermidades de maior peso na história da morbidade bucal ${ }^{1}$. Ela pode provocar limitações no cotidiano das pessoas ao desencadear dores e perdas dentárias. E isso contribui para gerar mudanças biopsicossociais que poderão interferir na qualidade de vida dos indivíduos.

0 termo qualidade de vida tem um conceito geral einclui a variedade de condições quepode rão afetar a percepção das pessoas, os sentimentos e os comportamentos. Nesse panorama, pesquisadores da Organização Mundial de Saúde (OM S) definiram qualidade de vida como sendo "a percepção do indivíduo de sua posição na vida no contexto da cultura e sistema de valores nos quais ele vive e em relação aos seus objetivos, expectativas, padrões e preocupações"2. A avaliação de qualidade de vida leva em consideração não apenas a saúde física, mas, também, o estado psicológico, o nível de independência, os relacionamentos sociais, os fatores do meio ambiente e as crenças pessoais ${ }^{3}$.

0 debate sobre a relação entre saúde e qualidade de vida demonstra que esta se trata de uma representação social baseada em parâmetros subjetivos, como bem-estar e felicidade, e objetivos com base nas necessidades de uma determinada população ${ }^{4}$. Revisão sistemática sobre 0 tema qualidade de vida, a partir do banco de dados Scielo, constatou uma produção científica importante, com diversificação de abordagens e métodos, no período de 2001 a 20065. Contudo, ape sar de a mensuração de Qualidade de Vida ter sido também frequentemente utilizada no contexto de saúde bucal, detectou-se carência de estudos investigativos acerca do impacto da cárie dentária sobre a qualidade de vida em adultos.

Em busca realizada, em agosto de 2012, na base de dados Pubmed com limites para a idade adulta 19 anos e mais, sem limites para data de publicação e língua e utilizando os descritores ("quality of life" [M eSH Terms] OR ("quality"[All Fields] AND "life"[All Fields]) OR "quality of life"[All Fields]) AND "dental caries"[M eSH Terms] AND "adult"[M eSH Terms] foram encontrados 119 trabal hos. A maior parte dos estudos enfocou idosos, crianças e pessoas com condições sistêmicas específicas. A penas onze estudos visaram os adultos e trataram de qualidade de vida e saúde bucal6-16, sendo que nenhum destes utilizaram na metodologia o instrumento dequalidade de vida denominado WHOQOL-bref ${ }^{17}$, desenvolvido pelo Grupo de Qualidade de Vida da Organização M undial de Saúde (OM S). Trata-se de um instrumento curto, mas com características psicométricas satisfatórias ${ }^{18}$.

Tem-se quea utilização de indicadores subje tivos contribua para complementar as informações clínicas além de fornecer subsídios para conhecer as percepções do indivíduo acerca de sua condição bucal. Em adição, os indicadores subjetivos poderão ajudar na formulação de programas e serviços de saúde para melhorar a qualidade de vida das pessoas ${ }^{19}$, acometidas pela doença cárie dentária, considerada como problema de saúde pública no Brasil.

0 levantamento epidemiológico nacional, de 2010, identificou nos adultos brasileiros de 35-44 anos um número médio de dentes cariados cavitados igual a 1,48 dentes. N esse mesmo levantamento brasileiro, apenas 0,9\% dos adultos eram livres do acometimento da cárie, tanto no passado, representado pelas restaurações e extrações, como no presente, identificado pela presença de dente com cárie cavitada ${ }^{20}$. Isso caracteriza a alta prevalência da doença nosadultos brasileiros, sendo importante investigar sua relação com a qualidade de vida. A hipótese deste estudo é que a autopercepção negativa da qualidade de vida, medida pelo WHOQOL-bref, esteja associada à maior gravidade da doença cárie e presença de dente com cárie cavitada. N esse sentido, o objetivo desteartigo foi avaliar o impacto da cáriedentária na qualidade de vida de adultos residentes no entorno de Belo Horizonte, M inas Gerais, Brasil.

\section{Métodos}

Este artigo é oriundo de estudo aprovado pelo Comitêde Ética em Pesquisa da UniversidadeFe deral de M inas Gerais (COEP/U FM G). Trata-se de pesquisa quantitativa de caráter transversal envolvendo coleta de dados em domicílios. A população do estudo foi composta de adultos com idades compreendidas entre 35 a 44 anos, residentes na zona urbana demunicípios no entorno de Belo Horizonte, M inas Gerais, região caracterizada como a terceira maior aglomeração urbana do Brasil, com uma população composta por 4.882.977 habitantes, conforme o Censo 2010.

A amostragem foi por conglomerados ${ }^{21}$, considerando o porte populacional municipal, conforme detal hado em publicação prévia'a22. D evido ao método de amostragem por conglomerados considerou-se o deff $=2^{23}$, a fim de se corrigir a perda de variabilidade dos indivíduos obtendo- 
se, assim, uma amostra de 1.150 indivíduos com alocação proporcional em cada município sorteado. A seleção da amostra foi pela técnica de amostragem probabilística por conglomerados por estágios ${ }^{23}$.

O exameclínico decárie dentária contou com a partici pação de 1138 adultos, que consentiram com a realização do exame da cavidade bucal. 0 índice adotado para o levantamento da cárie dentária foi o CPOD, que representa o número total de dentes cariados, perdidos e obturados. 0 exame foi realizado por cinco cirurgiãs dentistas calibradas ${ }^{24}$, com resultados de concordância inter e intraexaminadoras, Kappa de 0,81 a 0,92, e de 0,80 a 1,00, respectivamente. Para fins de análise deste estudo, os participantes foram divididos em dois grupos de acordo com os seguintes critérios: presença de pelo menos um dente com cárie cavitada $(C>1)$ e alta gravidade da doença cárie dentária ( $C P O D>25)$, definida a partir do Significant Caries Index ( $\mathrm{SiC}$ ), índice proposto em 2000 por Bratthall ${ }^{25}$. O SiC permite verificar os casos de distribuição assimétrica, que corresponde à média do CPOD para um terço do grupo com os maiores níveis da doença.

Enquanto a presença de cárie cavitada mede a doença atual, a gravidade mede a história da doença ao longo do tempo, já que o CPOD considera também os desfechos da cárie, dentes perdidos e restaurados. Nesse sentido, para a pre valência da cárie dentária considerou-se apenas os indivíduos com pelo menos um dente presente na cavidade bucal (1104), já para a gravidade de cárie dentária considerou-se todos os examinados, dentados e edêntulos (1138).

Além do exame da cavidade bucal aplicou-se um questionário para o levantamento de dados socioeconômicos edemográficos, além do Inventário de Qualidade de Vida WH OQOL-bref, versão abreviada em português do Brasil (Quadro 1), disponibilizada pelo grupo da Organização Mundial de Saúde ${ }^{18}$. O WH O Q O L-bref é constituído de 26 questões, cada qual com cinco alternativas de resposta pela escala de Likert. 0 instrumento é composto por quatro domínios: físico, psicológico, relações social e meio ambiente, além de questões que avaliam a qualidade de vida no geral ${ }^{18}$.

Tanto os domínios quanto a QV geral são medidos em direção positiva, ou seja, os escores mais altos denotam uma melhor QV. Para esse cálculo, utilizou-se a sintaxe do software SPSS (Statistical Package for Social Science) que automaticamentechecou, recodificou eestabeleceu os escores dos domínios e das questões de QV em geral ${ }^{17}$, optando-se por uma escala de 0 a 20. Para a análise dos dados, comparou-sea QV dos adultos com a condição de saúde bucal.

Neste estudo, realizou-se uma análise estatística descritiva dos dados sociodemográficos e clínicos, pelo cálculo das médias, desvios padrão edas proporções. A análiseinferencial adotou os seguintes procedimentos estatísticos: teste t de Student e o coeficiente de correlação linear de Pearson, com significância assumida de $p<0,05$. Todo o tratamento estatístico foi realizado no SPSS 18.0. 0 peso de amostragem foi utilizado para compensar as probabilidades desiguais dos elementos da amostra ${ }^{26}$, sendo os resultados apresentados por meio de valores ponderados, tanto para os percentuais como para as médias.

\section{Resultados}

Entre os indivíduos dentados (1104) a maioria apresentou ausência de dentes cariados cavitados (643). Já entre todos os examinados (1138), dentados ou não, a maioria apresentou gravidade de cárie menor que 25 dentes comprometidos pela doença (1006). A Tabela 1 apresenta a distribuição percentual ponderada das características socioeconômicas e demográficas dosadultosconforme a prevalência e a gravidade da doença cárie dentária.

A Tabela 2 apresenta os escores médios, desvio padrão, domínios e qualidade de vida geral do WHOQOL-bref da população conforme a prevalência e gravidade da doença cárie dentária. Para o grupo que apresentou pelo menos um dentecom cárie dentária verificou-semédias crescentes para os domínios meio ambiente, físico, psicológico erelações sociais. Asmédias (desvios padrão) da qualidade devida (QV) geral foi igual a $14,65( \pm 2,88)$ para as pessoas com cárie dentária e $15,34( \pm 2,38)$ para aquelas com ausência de dente cariado ( $p<0,001)$.

No grupo de adultos com maior gravidade de cárie dentária a ordem crescente das médias dos domínios manteve a mesma encontrada no grupo de pessoas com cárie, meio ambiente, físico, psicológico e relações sociais. Para os adultos com maior gravidade de cárie dentária a média da QV geral foi igual a 14,33 $( \pm 3,16)$, inferior ao do grupo com menor gravidade da doença $(15,10$ $\pm 2,57)(p<0,001)$.

0 total de dentes com cárie cavitada entre 0 grupo de pessoas com pelo menos um dente cariado ( $\mathrm{N}=461$ ) variou de 1 a 19 , sendo a média igual a 3,03 ( $\pm 2,788)$, a mediana dois dentes ca- 
Quadro 1. Dimensões conceituais e itens de qualidade de vida. Versão em português do instrumento de avaliação de qualidade de vida (WHOQOL-bref).

\begin{tabular}{|l|l|}
\hline $\begin{array}{c}\text { Dimensão } \\
\text { Conceitual: }\end{array}$ & \\
\hline Físico & $\begin{array}{l}\text { Emín que medida você acha que sua dor (física) impede você de fazer o que você precisa? } \\
\text { O quanto você precisa de algum tratamento médico para levar sua vida diária? } \\
\text { Você tem energia suficiente para seu dia-a-dia? } \\
\text { Quão bem você é capaz de se locomover? } \\
\text { Quão satisfeito(a) você está com o seu sono? } \\
\text { Quão satisfeito(a) você está com sua capacidade de desempenhar as atividades do seu } \\
\text { dia-a-dia? } \\
\text { Quão satisfeito(a) você está com sua capacidade para o trabalho? }\end{array}$ \\
\hline Psicológico & $\begin{array}{l}\text { O quanto você aproveita a vida? } \\
\text { Em que medida você acha que a sua vida tem sentido? } \\
\text { O quanto você consegue se concentrar? } \\
\text { Você é capaz de aceitar sua aparência física? } \\
\text { Quão satisfeito(a) você está consigo mesmo? } \\
\text { Com que frequência você tem sentimentos negativos tais como mau humor, desespero, } \\
\text { ansiedade, depressão? }\end{array}$ \\
\hline $\begin{array}{l}\text { Qualidade de } \\
\text { vida geral }\end{array}$ & $\begin{array}{l}\text { Como você avaliaria sua qualidade de vida? } \\
\text { Quão satisfeito(a) você está com a sua saúde? }\end{array}$ \\
\hline Melações sociais & $\begin{array}{l}\text { Quão satisfeito(a) você está com suas relações pessoais (amigos, parentes, conhecidos, } \\
\text { colegas)? } \\
\text { Quão satisfeito(a) você está com sua vida sexual? } \\
\text { Quão satisfeito(a) você está com o apoio que você recebe de seus amigos? }\end{array}$ \\
Ambiente & $\begin{array}{l}\text { Quão seguro(a) você se sente em sua vida diária? } \\
\text { Quão saudável é o seu ambiente físico (clima, barulho, poluição, atrativos)? } \\
\text { Você tem dinheiro suficiente para satisfazer suas necessidades? } \\
\text { Quão disponíveis para você estão as informações que precisa no seu dia-a-dia? } \\
\text { Em que medida você tem oportunidades de atividade de lazer? } \\
\text { Quão satisfeito(a) você está com as condições do local onde mora? } \\
\text { Quão satisfeito(a) você está com o seu meio de transporte? }\end{array}$ \\
\hline
\end{tabular}

riados e o percentil $75 \%$ foi igual a quatro dentes cariados.

Os resultados da correlação linear entre os domínios do WHOQOL-bref e o número dedentes cariados foram apresentados na Tabela 3. Os valores dos coeficientes de correlações $(r)$ foram negativos para três domínios: psicológico, relações sociais e o mei o ambiente. Também foi verificado valor negativo para a qualidade de vida geral das pessoas e o número de dentes cariados. 0 coeficiente de determinação $\left(r^{2}\right)$ para o número de dentes cariados e o domínio psicológico foi 0,98, o que significa que $98 \%$ da variação no número de dentes cariados é explicada pelo domínio psicológico ou vice-versa. Os coeficientes de determinação para os domínios relações sociais e meio ambiente foram 1,12 e 1,28, respectivamente, o que explica o número de dentes cariados e os referidos domínios.

O CPOD da população examinada variou de 0 a 32, sendo a média igual a 16,91 ( $\pm 6,892)$, bem menor queo SiC, quefoi igual a 24,68. Os resultados referentes ao quartil do CPOD total foram: $25 \%$ igual a $13,50 \%$ igual a 18 e $75 \%$ igual a 21.

A correlação linear de Pearson entreos domínios do WHOQ OL-bref e o valor total do CPOD dos adultos configurou-se com valores de $r$ ne gativos para a maioria dos domínios (psicológico, relações sociais e meio ambiente). Também para a qualidade de vida no geral o valor de $r$ foi 
Tabela 1. Distribuição percentual da população conforme prevalência e gravidade de cárie dentária. Adultos de 35-44 anos residentes no entorno de Belo Horizonte, Minas Gerais, Brasil, 2010.

\begin{tabular}{|c|c|c|c|c|}
\hline \multirow{3}{*}{ Variáveis } & \multicolumn{2}{|c|}{$\begin{array}{c}\text { Cárie dentária } \\
\text { (Dentados - N = 1104) }\end{array}$} & \multicolumn{2}{|c|}{$\begin{array}{c}\text { Gravidade da cárie } \\
\text { (Dentados e não - N = 1138) }\end{array}$} \\
\hline & $n=643$ & $n=461$ & $n=1006$ & $n=132$ \\
\hline & $\begin{array}{l}C=0 \\
(\%)^{*}\end{array}$ & $\begin{array}{l}C \geq 1 \\
(\%)^{*}\end{array}$ & $\begin{array}{c}\text { CPOD }<25 \\
(\%)^{*}\end{array}$ & $\begin{array}{c}\mathrm{CPOD} \geq 25 \\
(\%)^{*}\end{array}$ \\
\hline \multicolumn{5}{|l|}{ Sexo } \\
\hline M asculino & 60,0 & 40,0 & 89,0 & 11,0 \\
\hline Feminino & 57,8 & 42,2 & 87,6 & 12,4 \\
\hline \multicolumn{5}{|l|}{ Idade } \\
\hline 35-39 anos & 59,2 & 40,8 & 94,2 & 5,8 \\
\hline 40-44 anos & 57,8 & 42,2 & 81,4 & 18,6 \\
\hline \multicolumn{5}{|l|}{ Estado civil } \\
\hline Casado/amasiado & 60,7 & 39,3 & 88,0 & 12,0 \\
\hline Outros & 53,8 & 46,2 & 88,2 & 11,8 \\
\hline \multicolumn{5}{|l|}{ Escolaridade } \\
\hline N ão superior & 56,7 & 43,3 & 87,6 & 12,4 \\
\hline Superior & 78,8 & 21,2 & 92,6 & 7,4 \\
\hline \multicolumn{5}{|l|}{ Renda familiar mensal } \\
\hline$\leq \mathrm{U} \$ 300,00$ & 49,7 & 50,3 & 84,0 & 16,0 \\
\hline$>\mathrm{U} \$ 300,00$ & 60,5 & 39,5 & 88,9 & 11,1 \\
\hline \multicolumn{5}{|c|}{ Renda per capita mensal } \\
\hline$\leq \mathrm{U} \$ 300,00$ & 56,8 & 43,2 & 86,2 & 13,8 \\
\hline$>$ U $\$ 300,00$ & 63,5 & 36,5 & 93,3 & 6,7 \\
\hline
\end{tabular}

*Valores percentuais ponderados a partir dos pesos amostrais.

Tabela 2. Escores médios e desvio padrão dos domínios e qualidade de vida (QV) geral do WHOQOL-bref segundo a prevalência e gravidade da cárie dentária. Adultos de 35-44 anos residentes no entorno de Belo Horizonte, Minas Gerais, Brasil, 2010.

\begin{tabular}{|c|c|c|c|c|c|c|}
\hline \multirow[t]{2}{*}{$\begin{array}{l}\text { Domínios e qualidade } \\
\text { de vida (QV) geral }\end{array}$} & \multicolumn{2}{|c|}{$\begin{array}{l}\text { Prevalência de cárie } \\
\text { Média (Desvio Padrão)* }\end{array}$} & \multirow[b]{2}{*}{$p$ valor** } & \multicolumn{2}{|c|}{$\begin{array}{c}\text { Gravidade da cárie } \\
\text { (Dentados e não }-\mathrm{N}=1138 \text { ) }\end{array}$} & \multirow[b]{2}{*}{$p$ valor* } \\
\hline & $C=0$ & $C \geq 1$ & & CPOD $<25$ & $C P O D \geq 25$ & \\
\hline & $13,87( \pm 1,76)$ & $13,50( \pm 1,81)$ & $p<0,001$ & $13,72( \pm 1,80)$ & $13,62( \pm 1,94)$ & $p<0,001$ \\
\hline & & & & & & \\
\hline & & & & & & $p<$ \\
\hline & $13,04( \pm 2,46)$ & $12,18( \pm 2,54)$ & & $12,65( \pm 2,51)$ & $12,88( \pm 2,69)$ & $p<0,001$ \\
\hline QV Geral & $15,34( \pm 2,38)$ & $14,64( \pm 2,88)$ & $p<0,001$ & $15,10( \pm 2,57)$ & $14,33( \pm 3,16)$ & $p<0,001$ \\
\hline
\end{tabular}

*M édias (desvio padrões) ponderados a partir dos pesos amostrais. ${ }^{* *}$ Teste de hi pótese estatísticat de Student para as médias ponderadas.

negativo $(-0,090)$ (Tabela 4). I sso significa que enquanto o valor do CPOD aumenta o escore da qualidadedevida (QV) geral diminui ( $p<0,001$ ). Desse modo, o aumento da gravidade da cárie foi associado à menor qualidade de vida geral das pessoas.

0 coeficiente de determinação $\left(r^{2}\right)$ para 0 CPOD e QV geral foi 0,81, o que significa que $81 \%$ da variação no CPOD total é explicada pela
QV geral ou vice-versa. O domínio psicológico explica $26 \%$ da variação do CPOD ou vice-versa.

\section{Discussão}

N este estudo, a escolha da faixa etária de 35 a 44 anos deve-se ao fato de ser a que serve de índice para avaliar a saúde na população adulta ${ }^{24}$. A 
Tabela 3. Correlação linear entre a qualidade de vida e 0 total de dentes cariados. Adultos de 35-44 anos residentes no entorno de Belo Horizonte, Minas Gerais, Brasil, 2010.

\begin{tabular}{lrc}
\hline \multicolumn{1}{c}{ Domínios } & \multicolumn{2}{c}{ Número de dentes cariados } \\
\cline { 2 - 3 } & $\mathrm{r}$ & $\mathrm{p}$ \\
\hline Físico & 0,046 & $\mathrm{p}<0,001$ \\
Psicológico & $-0,099$ & $\mathrm{p}<0,001$ \\
Relações Sociais & $-0,106$ & $\mathrm{p}<0,001$ \\
M eio Ambiente & $-0,113$ & $\mathrm{p}<0,001$ \\
QV Geral & $-0,032$ & $\mathrm{p}<0,001$ \\
\hline
\end{tabular}

Tabela 4. Correlação linear de Pearson entre os domínios e a qualidade de vida geral do WHOQOLbref e o CPOD total da população adulta ( $\mathrm{N}=$ 1138). Adultos de 35-44 anos residentes no entorno de Belo Horizonte, Minas Gerais, Brasil, 2010.

\begin{tabular}{lrc}
\hline \multirow{2}{*}{ Domínios } & \multicolumn{2}{c}{ CPOD } \\
\cline { 2 - 3 } & \multicolumn{1}{c}{$r$} & $p$ \\
\hline Físico & 0,001 & $p<0,001$ \\
Psicológico & $-0,051$ & $p<0,001$ \\
Relações Sociais & $-0,011$ & $p<0,001$ \\
M eio Ambiente & $-0,027$ & $p<0,001$ \\
QV Geral & $-0,090$ & $p<0,001$ \\
\hline
\end{tabular}

presença de dente cariado cavitado foi mais pre val ente entre as pessoas do sexo feminino, de 40 a 44 anos, sem escolaridade superior e de baixa renda familiar e per capita mensal.

Embora 0 atual estudo não tenha tido a intenção de incluir na análise a associação de características sociais, econômicas e demográficas com qualidade de vida e condição de saúde bucal, foi constatado em outro estudo, que objetivou revisar sistematicamente a literatura para evidenciar a associação entre parâmetros sociais e dimensão de impacto de saúde bucal na qualidade de vida das pessoas, que as condi ções sociais mais claramente associadas à percepção de impactos negativos da saúde bucal na qualidade de vida foram mulheres, de baixa escolaridade e baixa renda, além de imigrantes ou grupos étnicos minoritários ${ }^{27}$.

Para a gravidade de cárie dentária era de se esperar que o maior número de pessoas estivesse no grupo de menor gravidade, uma vez que o corte foi pelo SiC, que se refere ao CPOD médio de cerca de um terço da população mais afetada pela doença. Tickle ${ }^{28}$ reconheceu a concentração de maior carga de doença em pequenos grupos populacionais, como fenômeno de polarização.

Um dado positivo encontrado neste estudo trata-se da constatação da maioria dos adultos apresentar ausência de dentes cariados. Para explicar esse resultado, tem-se como hipótese quea população adulta, do entorno de Belo H orizonte, não apresenta dificuldade de acesso aos serviços odontológicos. Nesse sentido, no Brasil, a pesquisa do IBGE em 2008 apontou redução no contingente populacional sem acesso ao dentista, re duzindo o percentual de $18,7 \%$ para $11,6 \%$, num período de 10 anos $^{29}$. Ademais, observou-se, no período decorrido entre 2003 a 2008, um maior empenho do governo no fortal ecimento de ações de monitoramento e de avaliação na atenção primária à saúde (APS), além do aumento de valores per capita dos recursos federais para a APS ${ }^{30}$.

A média do $\operatorname{CPOD}(16,8)$ foi semelhante à média encontrada no Levantamento Epidemiológico de Base Nacional de 2010 (16,3) para osadultos com a mesma faixa etária do atual estudo ${ }^{20}$.

Os adultos do grupo com presença de dentes cariados apresentaram escores médios do WHOQ O L-bref entre 12,18 a 15,77, o que significa de $60,95 \%$ a $78,55 \%$ da possibilidadedeseal cançar a maior qualidade de vida nos diferentes domínios. Já no grupo de adultos com maior gravidade de cáriedentária ( $C P O D \geq 25$ ), os escores médios de qualidade devida variaram de 12,65 a 15,41 ou de $63,25 \%$ a $77,05 \%$ da mais alta qualidade de vida.

As médias dos domínios e da qualidade de vida (QV) geral do WHOQ OL-bref foram maiores nos grupos com melhores condições de saúdebucal, ou seja, naqueles com ausência de dente cariado enos com menor gravidade de cáriedentária. N esse sentido, a ausência de cárie e o me nor CPOD foram associados a uma melhor qualidade de vida $(p<0,001)$.

O número de dentes com cárie por pessoa variou de 1 a 19, no entanto ao analisar o percentil $75 \%$, que foi igual a quatro dentes cariados, confirma-se o fenômeno da polarização. Este, estaria relacionado à noção de que em um polo há ausência da doença e, no outro, um maior número de casos concentrados em pequeno grupo populacional ${ }^{28,31}$. Esse resultado reforça a importancia de avaliar a gravidade da cárie dentária baseado no parâmetro do Sic, como adotado neste estudo.

Tanto na população de pessoas sem dente cariado como naquela com menor gravidade de cárie o domínio relações sociais apresentou as 
melhoresmédias, 15,77 e 15,41, respectivamente. Desse modo, o suporte social, percebido e recebido deamigos, foi relacionado à melhor condição de saúde bucal. Conforme Plazaola-Castano et al. ${ }^{32}$ o apoio social trata-se de um constructo multidimensional que pode ser definido como o grau que as necessidades básicas da população são satisfeitas por meio de interações interpessoais, podendo o apoio ser tanto no emocional quanto no instrumental.

O domínio relações sociais do WH OQOL-bref diz respeito à satisfação com as relações pessoais, com a vida sexual e com o apoio que recebe de amigos. N esse sentido, o maior cuidado com a saúde bucal, ausência de dente cariado cavitado e menor gravidade da doença cárie dentária, pode ter melhorado as relações pessoais e a qualidade de vida autopercebida.

No que se refere à correlação linear entre os domínios do WHOQOL-bref eo número de dentes cariados, os valores dos coeficientes das correlações apresentaram-se negativos para os três domínios: psicológico, relações sociais, meio ambiente e também para a qualidade de vida geral das pessoas. Esses resultados reforçam que 0 maior número de dentes cariados cavitados poderá influenciar, desfavoravelmente, na autopercepção da qualidade de vida entre os adultos.

N este estudo, foi observado que, quase a totalidade (98\%) da variação no número de dentes cariadoséexplicada pelo domínio psicológico ou vice versa, no qual as questões abordam os itens de capacidade de concentração, de aceitação da aparência física e de satisfação interior, entre outros. Sabe se que a cárie poderá ter como desfecho a dor de dente que poderá interferir na capacidade de concentração. Além disso, comumente, deixa marcas nos dentes ou pode destruir a estrutura dentária levando a perdas e esses desfechos poderão interferir na aceitação da própria aparência física e na satisfação do indivíduo com ele próprio.

Assim, os agravos que acometem a cavidade bucal afetam diretamente a capacidade mastigatória, a fala e acarretam sofrimento devido à dor frequentemente associada. Em adição, podem afetar a autoestima e o bem-estar geral dos indivíduos $^{33,34}$. Portanto, a qualidade de vida é fortemente influenciada pela sua condição de saúde bucal. As restrições, causadas pelas doenças bucais, nos campos físicos e psicológicos poderão influenciar diretamente na alimentação, fala, locomoção, convívio social e na autoestima34.

O coeficiente de determinação para o domínio meio ambiente foi 1,28 para a explicação do número de dentes cariados. Itens abordadosnes- se domínio como ter dinheiro suficiente para satisfazer as necessidades, disponibilização de informações no dia-a-dia ea satisfação com o acesso aos serviços de saúde podem ter contribuído muito para a correlação negativa entre o domínio meio ambiente e o número de dentes cariados. Desse modo, a avaliação da qualidade de vida nesse domínio foi melhor para os adultos sem dentes cariados cavitadose para aquel es com menor gravidade da doença.

Estudos realizados no Canadá35 e na Inglaterra ${ }^{36}$ demonstraram que, nas áreas onde há falta de acesso a recursos materiais e participação comunitária, o contexto social repercutiu na saúde bucal de adultos. No Brasil, estudo comprovou que a iniquidade de renda municipal foi associada ao maior número de dentes cariados ${ }^{37}$, e que os fatores socioeconômicos influenciam o processo deadoecer ou deter saúde, entre as pessoas ${ }^{38}$. Em adultos, de municípios do entorno de Belo H orizonte, a maior gravidade de cárie dentária foi constatada entre aqueles com menor renda familiar mensal eentre os que residiam em regiões sem petições comunitárias aos líderes políticos ${ }^{22}$. Desse modo, a posição que cada grupo social ocupa na sociedade leva a um maior ou menor risco para as doenças e possibilita ou não 0 acesso aos serviços de saúde ${ }^{39}$.

Os domínios do W HO Q O L-bref eo valor total do CPOD dos adultos apresentaram correlação negativa aos domínios psicológico, relações sociais emeio ambiente, etambém à QV no geral. Pessoas com maior gravidade de cárie apresentaram menor qualidade de vida, sen do que $81 \%$ da variação no CPOD total é explicada pela QV geral.

0 presente estudo tem limitações como ter sido conduzido apenas em parte da população e do território do entorno da capital de Minas Gerais, Belo Horizonte. Entretanto, algumas medidas foram tomadas para tornar a amostra representativa da região estudada: agrupamento de municípios por porte populacional, correção da amostra pelo efeito do desenho (deff), sorteio aleatório das unidades amostrais e utilização de peso de expansão na análise estatística para compensar as probabilidades desiguais dos elementos da amostra, por se tratar de amostragem por conglomerados. Cabe ressaltar a possibilidade de subestimação do total de dentes cariados na população estudada, uma vez que ao exame visual as cáries ocultas e as interproximais podem não ser identificadas.

Outra limitação refere-se ao desenho do estudo, de cunho transversal. Todas as observações foram feitas em cada indivíduo, em uma 
única oportunidade, não sendo possível estabe lecer a precedência temporal das variáveis independentes sobre os desfechos estudados, presença de dente cariado cavitado e maior gravidade de cárie dentária. É importante ressaltar que os coeficientes de $r$ e $r^{2}$ são respectivamente medidas de associação linear e de determinação, não estabelecendo, contudo, relações de causalidade.

A pesar das limitações, esteestudo érelevante por ser o primeiro que avaliou as relações entrea qualidade de vida e a cárie dentária em adultos residentes em região metropolitana brasileira e por se tratar de uma temática pouco explorada nos adultos em geral.

Concluindo, os resultados deste estudo sugerem quea presença de dente cariado com cavitação e a maior gravidade de cárie dentária são condições que poderão proporcionar reflexos diferenciados no cotidiano das pessoas e interferir, negativamente, na autopercepção da qualidade de vida. Isso porque uma melhor qualida- de de vida foi verificada nas pessoas sem cárie dentária e naquelas com menor gravidade da doença. A relação existente entre a condição de saúde bucal e a qualidade de vida demonstrou ser importante considerar as dimensões sociais e psicossociais nos levantamentos de saúde bucal.

Nesse sentido, novos caminhos devem ser trilhados no campo da saúde coletiva, para entender a interface entre a doença cárie dentária e a menor qualidade de vida. A correlação negativa entre cárie e qualidade de vida sugere que a doença impacta negativamente na qualidade de vida de adultos. Os indicadores subjetivos qualificaram as informações clínicas de saúde bucal, pois o modo como cada indivíduo vive e se relaciona com a doença cárie dentária é único e pessoal, edependentedefatores como o perfil psicológico e as condições ambientais e sociais. 0 conhecimento multifacetado pode contribuir para subsidiar ações e fomentar intervenções adequadas de políticas de saúde bucal.

\section{Colaboradores}

SM Costa, M Vasconcellos, M HGN Abreu participaram de todas as etapas: concepção, delineamento, análise e interpretação dos dados, redação do artigo. MHGN Abreu orientou todas as fases do estudo.

\section{Agradecimentos}

À Fundação de Amparo à Pesquisa do Estado de $M$ inas Gerais (Fapemig) pela bolsa concedida à doutoranda SM Costa. 


\section{Referências}

1. Rihs LB, Sousa MLR, Wada RS. Prevalência de cárie radicular em adultos e idosos na região sudeste do Estado de São Paulo, Brasil. Cad Saude Publica 2005; 21(1):311-316.

2. The Whogol Group. The development of the World $\mathrm{H}$ ealth Organization quality of life assessment instrument (the WHOQOL). In: Orley J, Kuyken W, editors. Quality of life assessment: international perspectives. Heidelberg: Springer Verlag; 1994. p. 41-60.

3. Silva SRC, Valsecki Júnior A. Avaliação das condições de saúde bucal dos idosos em um município brasileiro. Rev Panam Salud Pública 2000; 8(4):268271.

4. M inayo M CS, Hartz ZM A, Buss PM. Qualidade de vida e saúde: um debate necessário. Cien Saude Colet 2000; 5(1):7-18.

5. Landeiro GM B, Pedrozo CCR, Gomes MJ, Oliveira ERA. Revisão sistemática dos estudos sobre qualidade de vida indexados na base de dados SciELO. Cien Saude Colet 2011; 16(10):4257-4266.

6. M ehrstedt M, Tonnies S, Eisentraut I. Dental Fears, Health Status, and Quality of Life. Anesth Prog 2004; 51(3):90-94.

7. Lacerda JT, Castilho EA, Calvo M CM, Freitas SFT. Oral health and daily performance in adults in Chapecó, Santa Catarina State, Brazil. Cad Saude Publica 2008; 24(8):1846-1858.

8. Herenia PL, W MT, Jonathan M B, Richie P. Oral health-related quality of life in a birth cohort of 32year olds. Community Dent Oral Epidemiol 2008; 36(4):305-316

9. Gomes AS, Abegg C, Fachel JMG. Relationship between oral clinical conditions and daily performances. Braz. oral res 2009; 23(1):76-81.

10. Cohen-Carneiro $F$, Rebelo $M A B$, Souza-Santos $R$, Ambrosano GM B, Salino AV, Pontes DG. Psychometric properties of the OHIP-14 and prevalence and severity of oral health impacts in a rural riverine population in Amazonas State, Brazil. Cad Saude Publica 2010; 26(6):1122-1130

11. Daly B, Newton T, Batchelor $P$, Jones $K$. Oral health care needs and oral health-related quality of life (OHIP-14) in homeless people. Community Dent Oral Epidemiol 2010; 38(2):136-144.

12. Andersson P, Hakeberg M, Karlberg G, Ostberg AL. Clinical correlates of oral impacts on daily performances. Int J Dent Hyg. 2010; 8(3):219-226.

13. Bandéca $M C$, Nadalin $M R$, Calixto $L R$, Saad JR, da Silva SR. Correlation between oral health perception and clinical factors in a Brazilian community. Community Dent Health 2011; 28(1):64-68.

14. Montero J, Bravo M, Vicente MP, Galindo MP, Lopez-Valverde A, Casals E, Cortes-M artinicorena FJ, Llodra JC. Oral pain and eating problems in Spanish adults and elderly in the Spanish National Survey performed in 2005. J Orofac Pain 2011; 25(2): 141-152.

15. Zaitsu $T$, U eno $M$, Shinada $K$, Ohara S, Wright FA, Kawaguchi Y. Association of clinical oral health status with self-rated oral health and $\mathrm{GOHAl}$ in Japanese adults. Community Dent $\mathrm{H}$ ealth 2011; 28(4):297-300.
16. Slade GD, Sanders AE. The paradox of better subjective oral health in older age. J Dent Res. 2011; 90(11):1279-1285.

17. World Health Organization. Division of Mental $\mathrm{H}$ ealth and Prevention of Substance Abuse. Programme on Mental Health. WHOQOL Group. WHOQOL: M easuring quality of life [serial on the Internet] 1997. [cited 2009 may 10]. Available from: http://www.who.int/en/

18. Fleck MPA, Louzada S, Xavier M, Chachamovich R, Vieira G, Santos L, Pinzon V. Aplicação da versão em português do instrumento abreviado de avaliação da qualidade de vida "WHOQOL-bref". Rev Saude Publica 2000; 34(2):178-183.

19. M iotto MHM B, Barcellos LA, Velten DB. Avaliação do impacto na qualidade de vida causado por problemas bucais na população adulta e idosa em município da Região Sudeste. Cien Saude Colet 2012; 17(2):397-405.

20. Brasil. Ministério da Saúde (MS). Secretaria de Vigilância em Saúde. Secretaria de Assistência à Saúde. Departamento de Atenção Básica. Coordenacão Nacional de Saúde Bucal. Pesquisa Nacional de Saúde Bucal - 2010. Resultados principais. Brasília: M S; 2011.

21. Levy P, Lemeshow S. Sampling for health professionals. Belmont: Lifetime Learning Publications; 1980.

22. Costa SM, Vasconcelos M, Haddad JPA, Abreu MHNG. The severity of dental caries in adults aged 35 to 44 years residing in the metropolitan area of a large city in Brazil: a cross-sectional study. BM C Oral Health 2012; 12:25.

23. Brasil. M inistério da Saúde (MS). Secretaria de Políticas de Saúde. Coordenação de Desenvolvimento de Práticas da Atenção Básica. Área Técnica de Saúde Bucal. Projeto SB2000: condições de saúde bucal da população brasileira no ano 2000 - manual do coordenador. Brasília: MS; 2001.

24. World Health Organization (WHO). Oral health surveys: basic methods. 4a Edição. Geneva: ORH, EPID; 1997.

25. Bratthall D. Introducing the Significant Caries Index together with a proposal for a new global oral health goal for 12-year-olds. Int Dent J 2000; 50(6):378-384.

26. Dargatz DA, Hill GW. Analysis of survey data. Preventive Veterinary M edicine 1996; 28(4):225-237.

27. Cohen-Carneiro F, Souza-Santos R, Rebelo MAB. Quality of life related to oral health: contribution from social factors. Cien Saude Colet 2011; 16(Supl. 1):1007-1015.

28. Tickle $M$. The 80:20 phenomenon: help or hindrance to planning caries prevention programmes? Community Dent $\mathrm{H}$ ealth 2002; 19(1):39-42.

29. Instituto Brasileiro de Geografia e Estatística (IBGE). Pesquisa Nacional por Amostra de Domicílios PNAD/Brasil. 2008. [acessado 2010 jul 23]. Disponível em: http://www.ibge.gov.br/home/estatistica/ populacao/panorama_saude_brasil_2003_2008/ defaulttab pdf 2 .shtm 
30. Castro ALB, Machado CV. A política de atenção primária à saúde no Brasil: notas sobre a regulação e o financiamento federal. Cad Saude Publica 2010; 26(4):693-705

31. Macek MD, Heller KE, Selwitz RH, Manz M C.Is 75 percent of dental caries really found in 25 percent of the population? J Public Health Dent 2004; 64(1):20-25.

32. Plazaola-Castano J, Ruiz-Perez I, M ontero-Pinar MI, Grupo de estudio para la violencia de genero. Apoyo social como factor protector frente a la violencia contra la mujer en la pareja. Gac Sanit 2008; 22(6):527-533.

33. Locker D. Deprivation and oral health: a review. Community Dent Oral Epidemiol 2000; 28(3):161-169.

34. Sheiham A, Steele JG, M arcenes W, Tsakos G, Finch $S$, Walls AWG. Prevalence of impacts of dental and oral disorders and their effects on eating among older people; a national survey in Great Britain. Community Dent Oral Epidemiol 2001; 29(3):195-203.

35. Locker D, Ford J. Using area-based measures of socio-economic status in dental health services research. J Public Health Dent 1996; 56(2):69-75.

36. Aggarwal V, Macfarlane T, Macfarlane G. Why is pain more common amongst people living in areas of low socio-economic status? A population-based cross-sectional study. Br Dent J 2003; 194(7):383-387.

37. Celeste RK, Fritzell J, Nadanovsky P. The relationship between levels of income inequality and dental caries and periodontal diseases. Cad Saude Publica 2011, 27(6):1111-1120.

38. Cimões R, Caldas Júnior AF, Souza EHA, Gusmão ES. Influência da classe social nas razões clínicas das perdas dentárias. Cien Saude Colet 2007; 12(6): 1691-1696.

39. Boing AF, Peres MA, Kovaleski DF, Zange SE, Antunes JLF. Estratificação sócio-econômica em estudos epidemiológicos de cárie dentária e doenças periodontais: características da produção na década de 90. Cad Saude Publica 2005; 21(3):673-678.

Artigo apresentado em 27/06/2012

Aprovado em 12/08/2012

Versão final apresentada em 29/08/2012 